\title{
UTILIZAÇÕES E INTERAÇÕES MEDICAMENTOSAS DE PRODUTOS CONTENDO O GINKGO BILOBA
}

\author{
Thiago Ferreira Oliveira da Silva ${ }^{1}$, Carlos Eduardo Marcelino ${ }^{1}$, Ana Julia Pereira Santinho Gomes ${ }^{2}$ \\ ${ }^{1}$ Farmacêutico e Especialista em Farmácia Magistral, ${ }^{2}$ Universidade Federal de São João Del-Rei - UFSJ.
}

\section{RESUMO}

O extrato de Ginkgo Biloba tem sido utilizado em diversas formas farmacêuticas como cápsulas, cremes, xampus e outras formas, tendo múltipla utilização para o tratamento de diversas patologias e sendo um dos medicamentos mais antigos utilizados e estudados até hoje. $\mathrm{O}$ objetivo deste trabalho foi realizar um levantamento bibliográfico sobre as principais utilizações deste fitoterápico, mostrando várias indicações terapêuticas, tais como vertigens, cefaléias e distúrbios circulatórios periféricos, entre outras doenças. Além disto, vale destacar seu papel como um medicamento e como tal suas contra-indicações como, por exemplo, crianças menores de 12 anos e pacientes em tratamento com anti-trombóticos. Seus efeitos adversos podem agravar o estado do indivíduo se não houver interrupção do tratamento. Soma-se a isto a necessidade de se fazer uma análise para minimizar o risco de possíveis interações medicamentosas antes do inicio do tratamento para que não ocorra, por exemplo, a interação entre Ginkgo e anti-hipertensivos inibidores dos canais de cálcio (nifedipina, anlodipina e diltiazem) o que pode aumentar os efeitos adversos destes fármacos.

Palavras-chave: Ginkgo Biloba, ginkgolídeos, plantas medicinais, interações de medicamentos.

\section{USES AND INTERACTIONS OF PRODUCTS CONTAINING GINKGO BILOBA}

\begin{abstract}
The extract of Ginkgo Biloba has been used in various dosage forms like capsules, creams, shampoos, and other forms, having multiple uses for the treatment of various diseases, one of the older drugs used and studied to date. The aim of this paper was to review the literature on the main uses of this herbal, showing various therapeutics uses, such as dizziness, headache, peripheral circulatory disorders and other diseases. Moreover, it is worth highlighting its role as a drug and as such its warnings, for example, children under 12 years and patients treated with antithrombotics. Its adverse effects may worsen the condition of the individual if there is not an interruption of treatment. Added to this the need to do an analysis to minimize the risk of possible drug interactions before starting treatment to avoid in example the interaction between Ginkgo and calcium channel inhibitors antihypertensives (nifedipine, amlodipine and diltiazem), that may increase adverse effects of these drugs.
\end{abstract}

Keywords: Ginkgo Biloba, ginkgolides, medicinal plants, drug interactions, herbal medicines. Phytotherapeutic Drugs. 


\section{INTRODUÇÃO}

Todo fitoterápico é obtido empregando-se exclusivamente matérias-primas ativas vegetais e é caracterizado pelo conhecimento da eficácia e dos riscos de seu uso, assim como pela reprodutibilidade e constância de sua qualidade (FERREIRA, 2008).

O Ginkgo Biloba é uma planta medicinal que tem sido estudada em diversas áreas das ciências, sendo a única representante viva do seu gênero, cuja origem remonta ao Paleozóico Superior (cerca de 250 milhões de anos) (SCHENKEL et al., 2000). Pertecente à família das Ginkgolaceae, o Ginkgo Biloba mostra grande resistência às agressões sofridas pelo meio ambiente. Após a incineração das árvores desta espécie, pela bomba atômica de Hiroshima em 1946, o Ginkgo foi a primeira espécie a brotar a partir das árvores originais. Resiste a insetos, micro-organismos e às toxinas ambientais das civilizações modernas, esta espécie habita a Terra por milhões de anos (LIMA; DAVID, 2006).

$O$ fruto tem aspecto carnoso e de cor amarela-esverdeada e esta planta se adapta aos mais variados e diferentes climas desde que não haja frio ou calor excessivos (ALONSO, 1998).

O Ginkgo parece ser a espécie viva de árvore mais antiga existente, e é uma das ervas medicinais mais vendidas na Europa e nos Estados Unidos, usada no tratamento de doenças cardiovasculares e do sistema nervoso central (THOMAS; THOMAS, 2004; CALIXTO, 2001).

\section{Extratos e composição química das folhas de Ginkgo}

Geralmente utilizado sob a forma de extrato, é constituído de numerosos componentes dessa folha. Como nem todos os componentes são úteis para a terapia, em 1965 foi introduzido na prática médica ocidental um extrato dessa planta, conhecido pelo nome de EGb 761 (LIMA; DAVID, 2006).
Dependendo do país de origem e da época da coleta, entre outros fatores, a porcentagem individual dos constituintes pode variar. Por este motivo, a utilização de um extrato padronizado é importante para a garantia do efeito como fitoterápico (DINATON, 2009).

A metodologia de obtenção de produtos derivados das folhas do Ginkgo conduz a fitoterápicos com diferentes composições, o que coloca em questão a aceitação da similaridade entre estes medicamentos, embora proveniente da mesma fonte vegetal (SCHENKEL et al., 2000).

Sob a denominação "extrato de Ginkgo" podem ser encontrados no mercado produtos secos obtidos a partir de extratos simples hidroalcoólicos, metanol ou etanol, ou extratos especiais, os quais são obtidos por meio de uma sequência bem especificada de passos tecnológicos de enriquecimento e purificação (SCHENKEL et al., 2000).

O extrato é preparado a partir de folhas de Ginkgo por meio de um complexo processo que remove o ácido gincólico, que é tóxico, fazendo com que haja redução do risco de reações alérgicas. Isto explica o maior numero de reações desta natureza quando as folhas são consumidas diretamente (THOMAS; THOMAS, 2004)

As folhas de Ginkgo contém dois grupos de compostos dotados de propriedades farmacológicas interessantes: os flavonóides e os diterpenos. Os flavonóides são representados por vinte compostos, entre eles derivados heterosídeos de flavonóis e biflavonóis. Aos diterpenos está relacionada a capacidade em inibir o fator de agregação plaquetária (PAF) e aos flavonóis é atribuída uma atividade captadora de radicais livres (SCHENKEL et al., 2000).

De acordo com a Lista de Medicamentos Fitoterápicos de Registro Simplificado, o extrato de Ginkgo deve conter Ginkgoflavonóies (22 a 
$27 \%$ ), determinados como quercetina, kaempferol e isorhamnetina; e terpenolactonas (5 a 7\%), determinadas como ginkgolídeos A, B, C, J e bilobalídeos (BRASIL, 2008).

\section{Farmacocinética}

Após a administração oral da solução ou comprimido contendo Ginkgo Biloba L., extrato seco, as terpenolactonas que compõem o ginkgolídeo A, ginkgolídeo $B$ e bilobalídeo apresentaram em humanos biodisponibilidade absoluta de $98 \%$ para o ginkgolídeo A, 79\% para o ginkgolídeo B e 72\% para o bilobalídeo. Após a administração de 80 mg de Ginkgo Biloba L., extrato seco, as concentrações plasmáticas máximas foram de $15 \mathrm{ng} / \mathrm{mL}$ para o ginkgolídeo A, $4 \mathrm{ng} / \mathrm{mL}$ para 0 ginkgolídeo $B$ e aproximadamente $12 \mathrm{ng} / \mathrm{mL}$ para o bilobalídeo. As meias-vidas foram de 3,9 horas (ginkgolídeo A), 7 horas (ginkgolídeo B) e 3,2 horas (bilobalídeo). Após a administração intravenosa as meias-vidas foram de 3,5 horas (ginkgolídeo A), 5,5 horas (ginkgolídeo B) e 3,2 horas (bilobalídeo). A ligação à proteína plasmática (sangue humano) é de $43 \%$ para o ginkgolídeo $A$, $47 \%$ para o ginkgolídeo B e $67 \%$ para o bilobalídeo (EQUITAM, 2007).

Estudos em ratos utilizando o EGb 761 marcados com radioisótopos demonstraram absorção de $60 \%$. As propriedades farmacocinéticas mostraram atender um modelo bicompartimental com aparente fase de primeira ordem e meia-vida de cerca de 4,5 horas. Durante as 3 primeiras horas, a radioatividade foi detectada principalmente no plasma, no entanto, após 48 horas, a atividade especifica dos eritrócitos foi a mesma do plasma (LIMA; DAVID, 2006).

\section{Farmacodinâmica}

Por se tratar de um extrato com vários componentes, o mecanismo de ação do Ginkgo biloba não pode ser totalmente esclarecido. As diversas frações isoladamente parecem ter seu papel. Ações aditivas, antagonistas e sinergísticas sugerem ocorrer em experimentos farmacológicos como resultado da interação de vários constituintes de diversos sítios de membrana. Foram identificados cinco tipos principais de ginkgolídeos assim designados: A, B, C, J e M. Estudos demonstraram que eles possuem atividade como antagonista das ações de potentes autacóides. A fração flavonóica provavelmente interfere com a fosfodiesterase da guanosina monofosfato (GMP), inibe a catecol-Ometiltransferase (COMT) e a monoaminoxidase (MAO). Estudos experimentais em coelhos demonstraram ação antitrombótica, aumentando a produção local de prostaglandina $\left(\mathrm{PGI}_{2}\right)$. Há também indícios de redução da permeabilidade capilar pela rutina presente no extrato (LIMA; DAVID, 2006).

\section{Utilização do Ginkgo}

O extrato das folhas de Ginkgo é indicado para transtornos cognitivos, incluindo demência e insuficiência vascular cerebral, perda de memória recente, cefaléia, vertigens e zumbidos (tinidos), além de instabilidade emocional com ansiedade. Pacientes com mal de Alzheimer podem apresentar uma modesta melhora no desempenho cognitivo e social (CLAYTON; STOCK, 2006; THOMAS; THOMAS, 2004).

De acordo com Fernandes (2005), o Ginkgo foi estudado como opção terapêutica nas demências do tipo Alzheimer e multi-infarto. Os resultados de uma meta-análise de quatro ensaios clínicos mostraram que 0 extrato padronizado de Ginkgo EGb 761 produziu um efeito positivo significativo sobre a função cognitiva em pacientes com doença de Alzheimer. Esses resultados foram comparáveis aos do donepezil, um inibidor da colinesterase utilizado no tratamento desta doença. Soma-se a isto, 
outro estudo envolvendo 50 pacientes com doença de Alzheimer, demonstrou uma melhora da sociabilidade, da depressão, da distração e do esquecimento (LIMA \& DAVID, 2006).

Outras indicações incluem distúrbios circulatórios periféricos, como exemplo, a melhoria da claudicação intermitente, melhoria da disfunção erétil secundária a terapias antidepressivas, aumento do fluxo sanguíneo periférico em pacientes com diabetes melito e melhoria na audição em pacientes, cuja audição está prejudicada secundariamente pela má circulação nas orelhas (CLAYTON; STOCK, 2006).

Estudos demonstraram melhora na distância deambulada e diminuição da dor, confirmando a eficácia deste fitoterápico para claudicação intermitente.

Em uma metanálise, sugeriu-se que 0 Ginkgo foi mais eficaz em comparação à pentoxifilina no alívio dos sintomas da claudicação intermitente. Outros usos sugeridos seriam o tratamento da degeneração macular e síndrome pré-menstrual (KATZUNG, 2005; THOMAS; THOMAS, 2004).

Adicionalmente, foi demonstrado em modelos animais e alguns estudos em seres humanos que o Ginkgo aumenta o fluxo sanguíneo e reduz a viscosidade do sangue, sendo que o aumento de óxido nítrico endógeno pode ser o mecanismo (KATZUNG, 2005).

O Ginkgo tem também a função de agir sobre a pele, aumentando a resistência dos capilares (ação vasotônica), a microcirculação superficial e oxigenação dos tecidos. Previne a peroxidação lipídica causada pelos radicais livres, evitando o envelhecimento cutâneo (CUNHA et al., 2004)

Age aumentando a resistência e diminui a permeabilidade vascular. Tem ação vasodilatadora periférica e inibidora da agregação plaquetária (D'IPPOLITO et al., 2005).
Cremes e loções contendo extrato glicólico ou extrato de folhas são úteis em peles sensíveis. São estimulantes celulares em peles com problemas vasculares, diminuem 0 envelhecimento cutâneo e limitam a formação de rugas. Também possuem atividade sobre a celulite e o shampoo com extrato aquoso de folhas é utilizado para a diminuição da formação de caspa (CUNHA et al., 2004).

$\mathrm{Na}$ China, as sementes do Ginkgo são chamadas de bai gou e são consideradas eficazes nos meridianos da acupuntura dos pulmões e dos rins, onde são utilizadas para problemas de asma e tosses peitorais com fleuma espessa. Também possuem efeito tonificante no sistema urinário e são utilizadas para incontinência e urina excessiva (MNIMH, 2000).

Por fim, as folhas possuem propriedades adstringente, antifúngica e antibacteriana e são consideradas benéficas para o coração e os pulmões, sendo que a inalação do decocto das folhas é utilizada no tratamento da asma. Ainda, as folhas fervidas para o tratamento de frieiras e nos transtornos hemorroidais são utilizadas em forma de "banho de assento" (D'IPPOLITO et al., 2005; ALONSO, 1998).

\section{Contra-indicações}

Este fitoterápico está contra-indicado nos casos de gravidez, lactação, hipersensibilidade da pele ou do trato gastrointestinal e também em pacientes submetidos à terapia anti-trombótica. (FERREIRA, 2008; P. R. VADE-MÉCUM DE MEDICAMENTOS, 2004).

Também pode diminuir a fertilidade entre homens e mulheres e deve se evitado por casais que desejam ter filhos (THOMAS; THOMAS, 2004)

Não pode ser usado em crianças menores de 12 anos, visto que o Ginkgo Biloba não foi suficientemente investigado nos casos de humor depressivo e cefaléia não relacionados 
com síndromes demenciais. Apenas um estudo demonstrou pequenas quantidades de colchicina com um preparado de Ginkgo, e esse composto pode bloquear a divisão celular e provocar aborto. Não deve ser utilizado previamente em cirurgias do trato gastrointestinal e por pacientes que apresentam desordens hemorrágicas, ou de risco de hemorragia intracraniana (hipertensão arterial sistêmica e placas amilóides de diabetes senil), pois pode contribuir para ocorrência de hemorragias (EQUITAM, 2007; THOMAS; THOMAS, 2004).

\section{Reações adversas}

Raramente podem ocorrer distúrbios gastrointestinais, cefaléia e nos casos de predisposição alérgica, podem acontecer reações alérgicas cutâneas (hiperemia, edema, prurido), a possível reação de hipersensibilidade que consistem na ocorrência de espasmos, câimbras e, em casos de toxicidade aguda, atonia $e$ fraqueza, ou transtornos circulatórios (queda de pressão artéria, lipotimia, cefaléia). Em casos isolados foi observado sangramento após 0 tratamento de longa duração com ○ Ginkgo, contudo, durante os estudos clínicos específicos realizados com a administração diária de $240 \mathrm{mg}$ do extrato de Ginkgo, não foram observadas alterações nos parâmetros de coagulação (incluindo teste de tromboplastina parcial, teste de protrombina e tempo de sangramento). Está descrito na literatura um caso isolado de hematoma subdural, supostamente relacionado com a ingestão diária de $120 \mathrm{mg}$ do extrato de Ginkgo por um período superior a 2 anos (DINATON, 2009; P.R. VADE-MÉCUM DE MEDICAMENTOS, 2004).

As desordens hemorrágicas, em consequência da utilização crônica, ocorrem devido ao aumento do potencial hemorrágico, possivelmente associados com a redução da agregação plaquetária através da inibição do PAF
(Fator de Agregação Plaquetária) pelos componentes ginkgolídeos. (P.R. VADE-MÉCUM DE MEDICAMENTOS, 2004).

As sementes contém a toxina 4-Ometilpiridoxina responsável por perda da consciência e convulsões. Crianças que ingerem grande quantidade (> 50 unidades) de semente de Ginkgo não-cozidas apresentam convulsões, e consequentemente, existe alguma preocupação do uso de altas doses em pacientes com potencial de convulsão (D'IPPOLITO et al., 2005; THOMAS \& THOMAS, 2004).

Soma-se a isto, a ocorrência de dermatite se houver contato com os frutos. Isto porque a polpa dos frutos e a casca das sementes contêm ácido ginkgólico e bilobina, que são estruturalmente relacionados com uruchióis encontrados na hera venenosa, na casca da manga e na casca da castanha de caju (D'IPPOLITO et al., 2005; P.R. VADE-MÉCUM DE MEDICAMENTOS, 2004).

Altas doses de Ginkgo podem causar agitação branda, diarréia, náuseas, vômitos e vertigem. Porém, estes efeitos adversos podem ser minimizados pela lenta titulação da dose, aumentando enquanto ela for tolerada (CLAYTON; STOCK, 2006).

\section{Posologia}

As doses recomendadas se apresentam distintas, uma vez que para cada tipo de extrato há concentrações diferentes, sendo assim a posologia pode variar conforme sugerido por Ferreira (2008) abaixo:

- Extrato seco (24\% de ginkgoflavonóides): 40 a $80 \mathrm{mg}, 3$ vezes ao dia;

- Extrato seco 5:1: 0,5 a 1,5 g ao dia;

- Pó: 600 a 900 mg, em 3 doses.

- Extrato fluido (1:1): 0,5 mL, 3 vezes ao dia;

- Infusão a 10\% (folhas secas): 3 xícaras ao dia.

- Extrato glicólico: preparações cosméticas 5 a $10 \%$. 
Para o tratamento relacionado à função cognitiva, doses totais diárias de 120 a $240 \mathrm{mg}$ divididas em duas ou três doses de extratos de folha de Ginkgo são recomendadas. Para a claudicação intermitente, $240 \mathrm{mg}$ por dia seriam preferíveis (THOMAS \& THOMAS, 2004).

Em relação ao uso do Ginkgo para melhorar as funções cognitivas de indivíduos adultos saudáveis com menos de 60 anos, uma revisão sistemática demonstrou que os resultados disponíveis até o momento foram insuficientes e apontam para a falta de evidências clínicas que justifiquem a utilização dos extratos padronizados de Ginkgo com esta finalidade (FERNANDES, 2005).

\section{Interações medicamentosas}

Os polifenóis, como o Ginkgo Biloba, são encontrados na natureza na forma glicosídica. Para absorção intestinal, a solubilização dos flavonóides é considerada um pré-requisito, e os derivados glicosilados devem ser primeiro hidrolisados. Alguns componentes da alimentação, tais como fibras e etanol podem afetar a solubilidade e, consequentemente, a absorção dos flavonóides (WILHELM FILHO et al., 2001).

O Ginkgo pode potencializar a ação de agentes anti-trombóticos (antiplaquetários e anticoagulantes, incluindo a heparina e varfarina) e aumentar o risco de hemorragia intracerebral. A administração de Ginkgo e ticlodipina aumenta o tempo de hemorragia em $150 \%$ (P.R. VADEMÉCUM DE MEDICAMENTOS, 2004).

Outra classe farmacológica que pode apresentar interação com Ginkgo biloba é a dos anti-inflamatórios não-esteroidais, em particular o ácido acetilsalić́lico e o ibuprofeno, aumentando o risco de hemorragia. Após a administração de Ginkgo com acido acetilsalicílico houve relato de hifema espontânea (ALEXANDRE et al., 2008; EQUITAM, 2007).
A administração de Ginkgo associada com diuréticos tiazídicos (hidroclorotiazida) pode provocar, após uma semana, aumento da pressão sanguínea, porém este quadro pode ser revertido com a suspensão das duas medicações. Além disto, o Ginkgo administrado com antihipertensivos inibidores dos canais de cálcio (nifedipina, anlodipina e diltiazem), pode aumentar os efeitos adversos destes fármacos (ALEXANDRE et al., 2008; EQUITAM, 2007; GINKGO, 2008).

A utilização concomitante de Ginkgo 80 $\mathrm{mg}$, duas vezes ao dia, com trazodona $20 \mathrm{mg}$, duas vezes ao dia, pode resultar em coma no terceiro dia. A injeção intravenosa de $1 \mathrm{mg}$ de flumazenil pode resolver este quadro. Essa interação pode ser explicada pelo fato de que os flavonóides presentes no Ginkgo podem aumentar a atividade gabaérgica, devido ao efeito direto nos receptores benzodiazepínicos. Além disso, o Ginkgo pode aumentar a atividade da isoforma CYP34A do sistema microssomal P450, elevando a concentração plasmática do metabólito da trazodona (ALEXANDRE et al., 2008; P.R. VADE-MÉCUM DE MEDICAMENTOS, 2004).

Adicionalmente, a utilização do Ginkgo com omeprazol diminui a concentração plasmática e do efeito terapêutico deste fármaco. No caso da associação deste fitoterápico com os antipsicóticos haloperidol e olanzapina ocorre o inverso, aumentando o efeito terapêutico destes dois fármacos (ALEXANDRE et al., 2008).

Altas doses de Ginkgo podem diminuir a eficácia da terapia com anticonvulsivantes, como a carbamazepina e o ácido valpróico, no controle das convulsões (HAWKIN; EHRLICH, 2007).

Adicionalmente, a associação com antidepressivos como a fluoxetina, sertralina, paroxetina, e escitalopram pode causar síndrome de serotoninérgica. Esta condição é caracterizada 
pela rigidez, taquicardia, hipetermia, agitação e sudorese (HAWKIN; EHRLICH, 2007).

\section{CONCLUSÃO}

A utilização de medicamentos fitoterápicos tem aumentado muito nos últimos anos, principalmente em pacientes com algum tipo de doença crônica. Lembrando que o Ginkgo é um medicamento, ele deve fugir da visão, ainda vigente, de que os fitoterápicos são medicamentos naturais totalmente seguros sem contra indicações.

O Ginkgo se mostra eficiente para diversos tipos de patologias, porém a administração deste fármaco deve ser respeitada como a de qualquer outro medicamento, acompanhando a eficácia do tratamento da doença para a qual foi prescrita em cada individuo. Devem ser considerados os possíveis riscos de reações adversas, tanto na administração sozinha do Ginkgo, quanto na administração concomitante com outros fármacos que podem prejudicar o estilo de vida do usuário deste medicamento.

\section{REFERÊNCIAS}

Alexandre RF, Bagatini F, Simões CMO. Interações entre fármacos e medicamentos fitoterápicos à base de ginkgo ou ginseng. Revista Brasileira Farmacognosia 2008; 18(1): 117-26.

Alonso JR. Tratado de fitomedicina: bases clínicas y farmacológicas. Buenos Aires: ISIS Ediciones SRL; 1998. p.542-51.

Brasil. Ministério da Saúde. Agência Nacional de Vigilância Sanitária. Instruções Normativas ํo 5 de 11 de dezembro de 2008. D.O.U. 12 de dez. 2008. Disponível em: $\underline{\text { http://e- }}$ legis.anvisa.gov.br/leisref/public/ showAct.php?id=34477\&word=lista\%20de\%20me dicamentos\%20fitoterapicos
Calixto JB. Medicamentos fitoterápicos. In: Yunes RA, Calixto JB. Plantas medicinais sob a ótica da química medicinal moderna. Chapecó: Universidade do Oeste de Santa Catarina; 2001. p.297-315.

Clayton BD, Stock YN. Terapia com fitoterápicos e suplementos dietéticos. In: Clayton BD, Stock YN. Farmacologia na prática de enfermagem. 13.ed. Rio de Janeiro: Elsevier; 2006. p.767-82.

Cunha AP, Silva AP, Roque OR, Cunha E. Plantas e Produtos Vegetais em Cosmética e Dermatologia. Lisboa: Fundação Calouste Gulbenkian; 2004. p.161-2.

Dinaton. [bula de remédio na Internet]. Guarulhos: Aché [citado 2009 abr. 3]. Disponível em: http://www.ache.com.br/Research/pesquisainovacao-fito-dinaton-aspx

D'ippolito JAC, Rocha LM, Silva RF. Fitoterapia magistral: um guia prático para a manipulação de fitoterápicos. São Paulo: Anfarmag; 2005; p.91-4.

Equitam. Responsável técnico Sônia Albano Badaró. São Paulo: Eurofarma; 2007. Bula de remédio.

Fernandes AR, Nath GF, Simões CMO. Medicamentos fitoterápicos elaborados com Ginkgo, Hipérico, Kava e Valeriana. Acta Farmacológica Bonaerense, 2005; 24(2): 300-9.

Ferreira AO. Guia prático da farmácia magistral. 3.ed. São Paulo: Pharmabooks; 2008. v.1. p.373.

Ginkgo. Responsável técnico Anny M. Trintini: Colombo, PR: Herbarium Laboratórios Botânico; 2008. bula de remédio.

Hawkin EB, Ehrlich SD. Possible Interactions with: Ginkgo Biloba. [periódico eletrônico] 2007 [Acessado em 10 jun 2009]. Disponível em: http://www.umm.edu/altmed/articles/ginkgobiloba-000902.htm 
Katzung BG. Farmacologia básica \& clínica. 9.ed. Rio de Janeiro: Guanabara Koogan; 2005. p.9067.

Lima JP, David JM. Plantas medicinais. Fármacos derivados de plantas. In: Silva P. Farmacologia. 7.ed. Rio de Janeiro: Guanabara Koogan; 2006. p.148-59.

Mnimh PO. O guia completo das plantas medicinais. 2.ed. Porto: Civilização; 2000. p.69.

P.R. Vade-mécum de medicamentos. 9.ed. São Paulo: Soriak; 2004.

Schenkel EP, Gosman G, Petrovick PR. Produtos de Origem Vegetal e o Desenvolvimento de Medicamentos. In: Simões CMO, Schenkel EP, Gosman G, Mello JCP, Mentz LA, Petrovick PR. Farmacognosia da Planta ao Medicamento. Florianópolis: UFSC; 2000. p.291-310.

Thomas JA, Thomas JM. Fitoterápicos (Ervas Medicinais). In: Craig CR, Stiezel RE. Farmacologia moderna com aplicações clínicas. 3.ed. Rio de Janeiro: Guanabara Koogan; 2004. p.691-6.

Wilhelm Filho D, Silva EL, Boveris A. Flavonóides antioxidantes de plantas medicinais e alimentos: importância e perspectivas terapêuticas. In: Yunes RA, Calixto JB. Plantas medicinais sob a ótica da química medicinal moderna. Chapecó: Universidade do Oeste de Santa Catarina; 2001. p.317-34. 